\title{
Aero Engine Performance Monitoring Using Least Squares Regression and Spectral Clustering
}

\author{
Kunaal SAXENA ${ }^{\mathrm{a}, 1}$ and Manisha J NENE ${ }^{\mathrm{a}}$ \\ a Department of CSE, Defence Institute of Advanced Technology, Pune, Maharashtra
}

\begin{abstract}
Threshold-based flight data recorder analysis techniques have been widely used across the aerospace industry for fault detection and accident prevention. These techniques can detect pre-programmed events but fail to capture unknown patterns in the dataset. This research proposes a machine learning (ML) algorithm to analyze and detect unusual aero engine performance of a turboshaft engine mounted on a single engine rotorcraft. The performance is first modelled from an FDR dataset consisting of hundred flights, using least squares regression (LSR). A technique to scale the model by adding flight data from subsequent flights is thereafter discussed. Spectral Clustering is used for testing and validating the hypothesis derived from the regression model, by employing synthetically generated FDR data for twenty-five flights.
\end{abstract}

Keywords. Machine Learning, aero engine, FDR, Least Squares Regression, Spectral Clustering.

\section{Introduction}

\subsection{FDR Analysis}

The flight data recorder (FDR) is an integral part of all modern aircraft. It records various aircraft and aeroengine parameters every second during flying and stores it in the FDR database. The analysis of this data from the FDR, is termed as Flight Data Monitoring (FDM). The purpose of FDM is to monitor the performance of an aircraft accurately and predict its serviceability. Most aircraft employ a threshold-based fault detection system for the same, which reports an event as a fault only when the threshold for an FDR parameter is triggered.

\subsection{Problem Statement}

Multiple facets to performance monitoring of aeroengines such as unusual fuel consumption, unusual take off power, abnormal gas generator rotation speed etc. go unnoticed by the existing threshold-based analysis.

\footnotetext{
${ }^{1}$ Kunaal SAXENA, Department of CSE, Defence Institute of Advanced Technology, Pune Email: motto877@gmail.com.
} 
This is because the present FDM system analyzes FDR data only from a single flight at a time and is not able to observe any changes in the trend of the operating parameters, from previous flights. This study aims to overcome this limitation by utilizing ML techniques, as they are able to draw inferences from a large number of flights and thus capable of observing the minutest of changes in the trend of aircraft and aero engine performance.

\subsection{Literature Review}

Until now, FDR analysis has not utilized ML techniques on a comprehensive basis [1]. However, over the years, efforts have been made towards research in ML for FDM. Chunxiao Zhang et al. proposed the use of support vector machines (SVM) regression to monitor aero-engine health and condition by comparing flight data of 100 flights with an engine baseline model, in [2]. Li-Hua Ren et al. proposed a data-driven method for modelling the aero-engine aerodynamic performance by combining stochastic gradient descent with SVM in [3]. The studies undertaken in both [2] and [3] utilize SVM Regression, which suffers from a major drawback of higher computational burden because of the required constrained optimization programming [4].

Lishuai Li et al, in [5] have carried out great research in the field of cluster-based anomaly detection [6] for detecting abnormal flight operations, using the technique of density based spatial clustering of applications with noise. Julian Oehling et al. used local outlier probability detection method to discover anomalies in flight performance from FDR data and demonstrated scaling up the method for 1.2 million flights in [6]. Although both techniques discussed in [5] and [6] were made scalable to accommodate additional flight data, they utilized unsupervised learning, which only helps in identifying anomalies and local outliers, without modelling the actual performance of an aircraft.

According to a study undertaken by Clinton et al. in [7], 64 percent of fatal aviation accidents due to material failure are caused because of failure of the aeroengine. The study undertaken in this paper therefore focuses on monitoring the performance of an aero engine. Least squares regression supervised learning is used to model the performance of an aero engine installed on a rotorcraft, from an FDR dataset consisting of 100 flights. The learned model is then used to detect unusual aero engine performance from a set of 25 synthetically generated flight datasets, using spectral clustering. The term 'unusual' refers to an aero engine performance that does not adhere to the inferences drawn from the developed ML model. The remainder of this paper is structured as follows. Section 2 discusses the overall workflow adopted for the research. Section 3 describes the implementation of regression modelling to discover relationships between the engine related FDR parameters. Further, a method to scale the regression algorithm is discussed, followed by the anomaly detection technique used to detect unusual engine performance. Section 4 discusses the results obtained from the applied regression-based model and anomaly detection algorithm. Section 5 concludes.

\section{ML Workflow}

Figure 1 depicts the overall workflow for building a ML model for learning the normal aeroengine performance. The aircraft FDR data is obtained from historical 
database. The database consists of a set of dependent and independent features. Only the aeroengine related features are selected and passed through a feature selection algorithm, to identify the degree of correlation between them. The subject data is preprocessed using a feature scaling algorithm, to scale all features in the dataset, within a uniform range. Using ordinary least squares regression technique, the data is mined to produce a regression-based model. The subject model is then used on test flight datasets to detect anomalies.

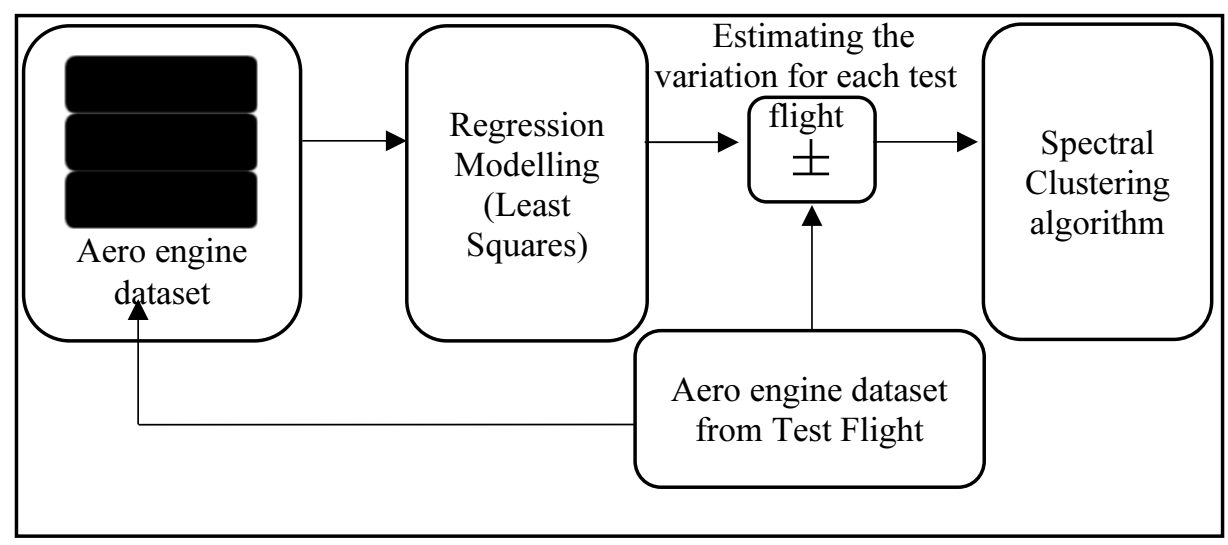

Figure 1. Workflow depicting the proposed ML technique

\section{Implementation of Regression Modelling and Anomaly Detection Algorithms}

\subsection{Regression modelling}

Aero-engine performance of turboshaft engines can be ascertained by the relative performance of some of its key parameters such as the gas generator turbine rotation speed (N1) and the generated torque (Q). Subject parameters depend upon the opening of the air flow inlet guide vanes $(\mathrm{GV})$, fuel Flow (FF) into the combustion chamber and the engine exhaust gas temperature (EGT). Thus, we get a set of dependent and independent features of the FDR dataset, which can be defined by the relationship: -

$$
\mathrm{y}=\mathrm{F}(\mathrm{x})+\mathrm{e}
$$

Here, ' $\mathrm{y}$ ' represents the set of dependent aeroengine features of N1 and Q, while ' $x$ ' represents the set of independent features GV, FF and EGT in the data set. The first objective of the regression problem is to identify the function $F$. In terms of a regression problem, we write,

$$
\mathrm{y}=\mathrm{F} \cdot \Phi(\mathrm{x})+£
$$


where, ' $\phi(x)$ ' represents a matrix of independent engine features, ' $F$ ' represents the matrix that defines the relationship between ' $y$ ' and ' $\phi(x)$ '. ' $£$ ' represents some noise which may be present in the FDR dataset, and is used as an error reducing coefficient for the regression overfit. Then, the matrices ' $y$ ' and ' $\phi(x)$ ' are constructed.

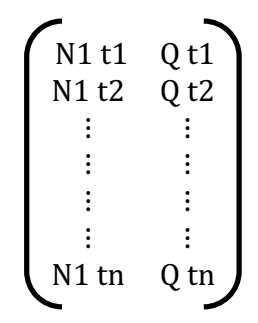

Matrix y $\operatorname{tn} \times 2$

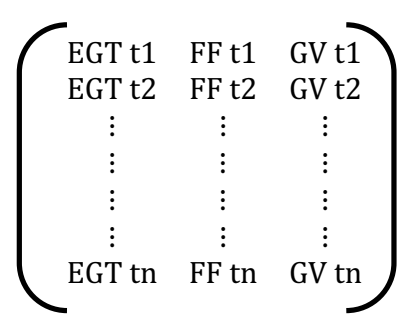

Matrix $\phi(x)$ $\operatorname{tn} \times 3$

The FDR dataset contains data for approximately 100 hours of flying, thus providing data for more than 3 lakh time samples, represented by the indices ' $t 1$ ' to ' $t n$ ' in the matrix. The solution for finding ' $\mathrm{F}$ ' is obtained using the regularized least squares approximation technique [8].

$$
\mathrm{F}=\operatorname{inv}\left[\left(\Phi(\mathrm{x})^{\mathrm{T}} \Phi(\mathrm{x})\right)+£\right] . \Phi(\mathrm{x})^{\mathrm{T}} \mathrm{y}
$$

The developed model ' $F$ ' models the performance of the aero engine i.e., it defines the true relationship between the dependent and independent features of the FDR dataset, with respect to the aero engine.

\subsection{Scaling for additional flight data}

The proposed technique is capable of handling large FDR data sets as well as continuously updating the model ' $\mathrm{F}$ ' with new flight data, by updating the matrices ' $\phi(\mathrm{x})$ ' and ' $\mathrm{y}$ '. Let ' $\mathrm{N}$ ' be the number of flights for which the model ' $\mathrm{F}$ ' was initially calculated and let and let ' $\mathrm{D}_{\mathrm{N}}$, be the number of data points for each of the ' $\mathrm{N}$ ' flights. Then, for the $(\mathrm{N}+1)^{\text {th }}$ flight, the model ' $\mathrm{F}$ ' needs to be updated with data points $\mathrm{D}_{(\mathrm{N}+1)}$ from the $(\mathrm{N}+1)^{\text {th }}$ flight. Updating the model with new flight dataset only requires the computation of the products as shown below, and then adding the obtained products to Eq. (3).

$$
\begin{aligned}
& \phi(\mathrm{x})_{(\mathrm{N}+1)}^{\mathrm{T}} \phi(\mathrm{x})_{(\mathrm{N}+1)} \\
& \phi(\mathrm{x})_{(\mathrm{N}+1)}{ }^{\mathrm{T}} \mathrm{y}_{(\mathrm{N}+1)}
\end{aligned}
$$

At each step, dataset of the new flight is added to the existing dataset by calculation of only the inner transpose products. In this way, the data processing technique is made scalable to include FDR datasets from all subsequent flights, without much additional computational overheads. This technique enables the derived 
regression model ' $\mathrm{F}$ ' to keep updating, correcting and tuning itself to the subsequent flights' aeroengine performance.

\subsection{Anomaly Detection}

Once the model ' $\mathrm{F}$ ' has been identified, the aim is to use it to discover anomalies in aero engine performance from test flight data. The anomaly detection algorithm also provides a way of evaluating the developed regression model. The subject test data has been generated synthetically and consists of a set of 10 flights with derated aeroengine performance and 15 flights showing normal performance. The first step is to find the variation ' $\mathrm{V}$ ' between each set of test flight data and trained data.

$$
\mathrm{V}_{\mathrm{i}}=\mathrm{y}_{\mathrm{i}}-\mathrm{F} \phi(\mathrm{x})_{\mathrm{i}}
$$

Here, $\mathrm{V}_{\mathrm{i}}$ represents the variation observed in the $\mathrm{i}^{\text {th }}$ test flight. Variations for all sets of test flight data are calculated sequentially. These are applied to a spectral clustering algorithm, enabling separating out the unusual aero engine performances, which are marked as outliers and require further inspection and investigation by aviation experts. Spectral clustering uses the connectivity approach to clustering, wherein communities of nodes (i.e. data points) that are connected are identified in a graph. These data points are then mapped to a low-dimensional space that are segregated to form clusters [9]. A detailed explanation of the spectral clustering algorithm, however, is beyond the scope of this paper.

\section{Results and Discussions}

The FDR dataset consists of the multiple sets of features, out of which the engine related features include N1, Q, GV, FF, EGT, and some general features like altitude (Alt) and the indicated air speed (IAS). The right set of features to be utilized for regression modelling were selected using the Spearman's rank correlation coefficient.

The features GV, EGT and FF showed a high degree of correlation $(>0.6)$ with the dependent features Q and N1 of the FDR dataset. Therefore, those features have been chosen as independent and dependent set of features, respectively. Regression modelling for the selected features yields the matrix ' $F$ ' of the order of $3 \times 2$.

Table 1. Regression Model Parameters (Matrix 'F')

\begin{tabular}{lll}
\hline & Q & N1 \\
\hline GV & 0.96534358 & 0.7342996 \\
\hline FF & 0.34389898 & 0.61503091 \\
\hline EGT & -0.31262837 & -0.3499808 \\
\hline
\end{tabular}




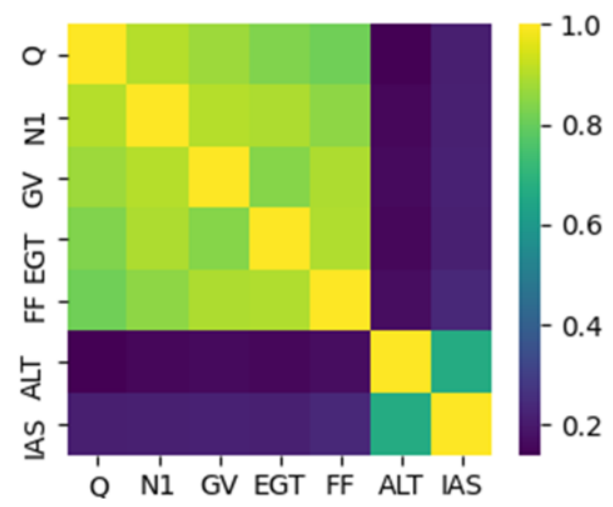

Figure 2. Correlation between FDR dataset features

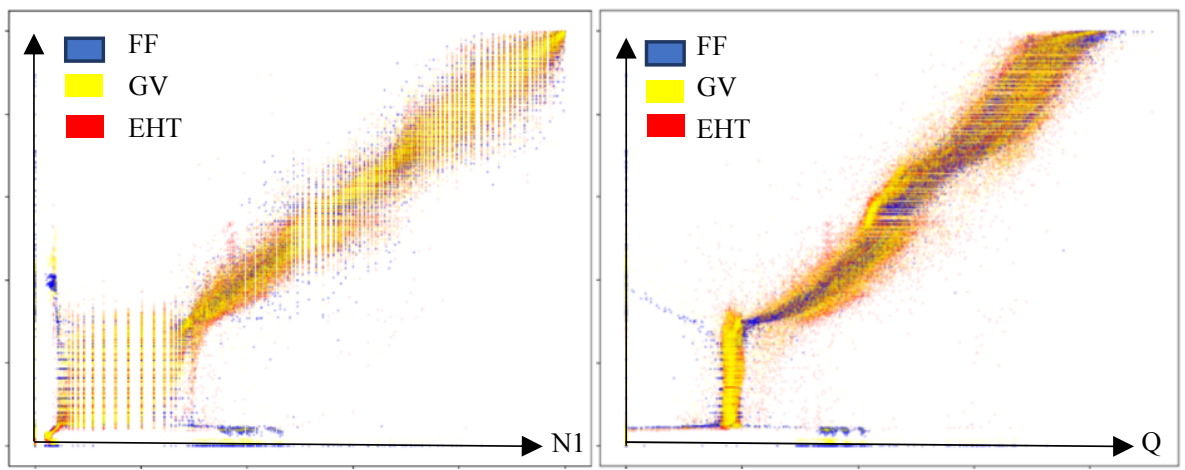

Figure 3. Linear relationship of $\mathrm{N} 1$ and $\mathrm{Q}$ with engine parameters FF, GV and EHT
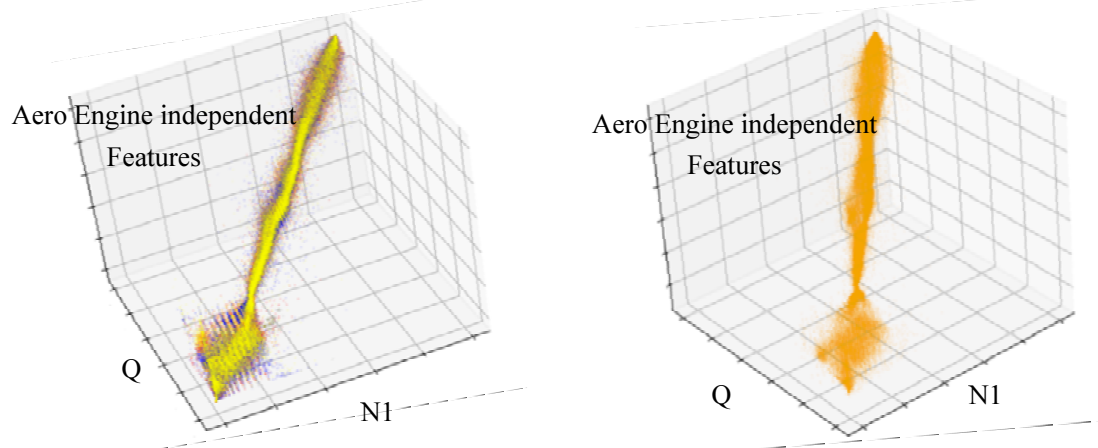

Figure 4. 3D plot showing relationship of $Q$ and N1 with independent aero engine features GV, FF and EGT and the developed regression model on the right.

A set of twenty-five Test Flight datasets were applied to the regression model. Variations from the model were clustered using Spectral Clustering, which yielded two sets of clusters, containing 10 flights and 15 flights, respectively. The two clusters represent unusual and normal aero engine performance in the test flights. 


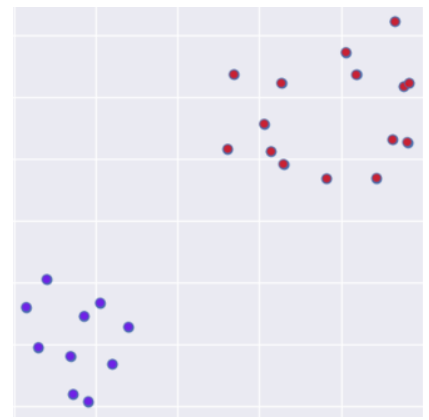

Figure 5. Spectral Clustering for 25 Test Flights

The 10 flights identified as displaying unusual aero engine performance were compared with the traditional threshold-based exceedance detection system. The maximum values reached by $\mathrm{Q}$ and N1 during the entire flight duration were not sufficient to trigger the threshold.

Table 2. Comparison of identified unusual performance test flights (TF) with N1 and Q thresholds

\begin{tabular}{|c|c|c|c|c|c|c|c|c|c|c|c|}
\hline & TF1 & TF2 & TF3 & TF4 & TF5 & TF6 & TF7 & TF8 & TF9 & TF 10 & Threshold \\
\hline$\overline{\mathbf{Q}}$ & 40.8 & 44.3 & 46.9 & 51.4 & 53.7 & 55.5 & 60.8 & 67.3 & 72.2 & 77.4 & 80 \\
\hline N1 & 46.6 & 50.4 & 54.6 & 58.1 & 61.3 & 63.4 & 69.8 & 76.2 & 82.3 & 88.5 & 92 \\
\hline
\end{tabular}

\section{Conclusion}

The data processing approach proposed in this paper uses least squares regression to model the performance of a turboshaft engine installed on a rotorcraft from a set of 100 flights. The model is validated by employing spectral clustering to detect unusual engine performance in 25 test flights. Spectral clustering was able to treat every node/ data point in a graph independently, which enabled in effectively separating out dis similar test flight data sets. Using the synthetically generated test flight data, the discussed ML algorithm was able to identify 10 flights showing unusual or derated aeroengine performance. The maximum values attained by $\mathrm{Q}$ and $\mathrm{N} 1$ in all the identified unusual test flights were less than the threshold values and would have gone unnoticed by traditional threshold-based analysis. This data processing approach, however, does not aim to replace the existing threshold-based analysis. But it provides a different perspective to FDM, by utilizing ML for establishing true relationships between the different FDR features.

\section{References}

[1] Jasra, Sameer \& Gauci, Jason \& Muscat, Alan \& Valentino, Gianluca \& Zammit-Mangion, David \& Camilleri, Robert. Literature review of machine learning techniques to analyse flight data. AEGATS Conference. 2108 Oct. Toulouse, France.

[2] Chunxiao Zhang, Nan Wang. Aero-Engine Condition Monitoring Based on Support Vector Machine. Physics Procedia. 2012; Volume 24, Part B, Pages 1546-1552. https://doi.org/10.1016/j.phpro.2012.02.228. 
[3] Li-Hua Ren, Zhi-Feng Ye, Yong-Ping Zhao. A modeling method for aero-engine by combining stochastic gradient descent with support vector regression. Aerospace Science and Technology. 2020; Volume 99. https://doi.org/10.1016/j.ast.2020.105775.

[4] Wang, Haifeng \& Hu, Dejin. Comparison of SVM and LS-SVM for regression. International Conference on Neural Networks and Brain Proceedings. 2005; doi.org/10.1109/ICNNB.2005.1614615.

[5] Lishuai Li, Santanu Das, R. John Hansman, Rafael Palacios and Ashok N. Srivastava. Analysis of Flight Data Using Clustering Techniques for Detecting Abnormal Operations. Journal of Aerospace Information Systems. 2015; p. 587-598. doi:10.2514/1.I010329.

[6] Julian Oehling, David J. Barry, Using machine learning methods in airline flight data monitoring to generate new operational safety knowledge from existing data, Safety Science. 2019; Volume 114, Pages 89-104. https://doi.org/10.1016/j.ssci.2018.12.018.

[7] Clinton V. Oster, John S Strong, Zorn Kurt. Why Airplanes Crash: Causes of Accidents Worldwide. 51st Annual Transportation Research Forum. 2010. Doi: 10.22004/ag.econ.207282.

[8] Shawe-Taylor J, Cristianini N. Kernel Methods: An Overview. Kernel Methods for Pattern Analysis. Cambridge: Cambridge University Press; 2004. doi:10.1017/CBO9780511809682.

[9] D. Huang, C. Wang, J. Wu, J. Lai and C. Kwoh. Ultra-Scalable Spectral Clustering and Ensemble Clustering. IEEE Transactions on Knowledge and Data Engineering. 2020 Jun 01; vol. 32, no. 6: pp. 1212-1226. doi: 10.1109/TKDE.2019.2903410. 